\title{
LINEAR FREE VIBRATION OF GRAPHENE SHEETS WITH NANOPORE VIA AIFANTIS THEORY AND RITZ METHOD
}

\author{
Sima ZiaeE \\ Yasouj University, Mechanical Engineering Department, Yasouj, Iran \\ e-mail: ziaee@yu.ac.ir
}

This article aims to study the natural frequency of defective graphene sheets since the existence of cut-outs in plates may be essential on the basis of their desired functionality. A combination of the Aifantis theory and Kirchhoff thin plate hypothesis is used to derive governing equations of motion. The Ritz method is employed to derive discrete equations of motion. The molecular structural mechanics method is also employed to specify the effective length scale parameter. In the 'numerical results' Section, the effects of different parameters such as boundary conditions and diameter of the hole-to-side length ratio on the fundamental frequency of graphene sheets are studied.

Keywords: free vibration, defective graphene sheet, Aifantis theory, molecular structural mechanics

\section{Introduction}

Graphene sheets, the two-dimensional carbon nanostructure, amazingly exhibit the electrical, mechanical, thermal and optical properties (Jomehzadeh et al., 2015) that have been considered as a promising material for a wide range of applications (Lebedeva et al., 2012) such as composites, chemical sensors, ultra capacitors, transparent electrodes, photovoltaic cells, bio-devices (Zandiatashbar et al., 2014) and the gigahertz oscillator suggested based on the telescopic oscillation of graphene layers (Lebedeva et al., 2012). It is of great importance to simulate mechanical behavior of single/muti-layered graphene sheets accurately due to this wide scope of applications. One important topic which has recently received great attention is the study of free and forced vibration of graphene sheets used in nano-scale devices which may experience vibration.

Among different theoretical modelings used to simulate carbon nano-structures, continuum mechanics modeling and molecular structural mechanics (MSM) methods are the most popular ones employed to study the mechanical behavior of carbon nanostructures because their computational tasks are much more time-effective than different atomistic modeling such as classical molecular dynamics, tight-binding molecular dynamics and density functional theory (Li and Chou, 2003; Hu et al., 2007). The linear vibration of zigzag and armchair single-layered graphene sheets (SLGS) was studied by Sakhaee-Pour et al. (2008) via the MSM approach. They obtained fundamental frequencies and corresponding mode shapes for different boundary conditions and showed that the natural frequencies of SLGS are independent of chirality and aspect ratio (Sakhaee-pour et al., 2008), while the Hashemnia et al. study (2009) indicated that the fundamental frequency of SLGS with fixed-fixed or fixed-free end conditions decreased as the aspect ratio increased. They proposed the use of graphene sheets with a lower aspect ratio to prevent resonance and dynamic damage (Hashemnia et al., 2009). Gupta and Batra (2010) implemented MSM simulation to investigate the effect of pretention on the natural frequencies of SLGS. The comparison between the results obtained by the MSM method and those predicted by an equivalent linear elastic isotropic continuum model showed that there was a noticeable 
difference between the mode shapes corresponding to the several lowest frequencies of the SLGS and those of an equivalent linear elastic isotropic continuum model (Gupta and Batra, 2010). Sadeghi and Naghdabadi (2010) introduced a hybrid atomistic structural element to model the nonlinear behavior of SLGS. Their results verified with experimental observations demonstrated that natural frequencies predicted by nonlinear analysis were far higher than those obtained by the linear approach. Wang et al. (2013) proposed a pseudo beam model with 3-node beam element to simulate the modal behavior of the wrinkled SLGS by using the MSM method. They not only obtained the features of formation and evolution of wrinkles but also offered a prediction model to estimate the natural frequency of wrinkle SLGS (Wang et al., 2013).

The importance of incorporating the size effect into continuum mechanics, in order to investigate the mechanical behavior of micro- or nano-scale devices, is well known and higher-order continuum theories containing additional material constants have been developed to this end (Askes and Aifantis, 2011). Mindlin (1964) developed a theory of elasticity with microstructure in which strain energy was considered as a function of macroscopic strain, the difference between macroscopic and microscopic deformation and the gradient of the microscopic deformation. In this theory, the strain energy contained 16 constitutive coefficients in addition to Lame's constants. Mindlin wrote the kinetic energy density in terms of quantities at microscale and macroscale as well (Askes and Aifantis, 2011). Mindlin also simplified this theory and introduced three new versions which differed in the assumed relation between the microscopic deformation gradient and the macroscopic displacement (Mindlin, 1964; Askes and Aifantis, 2011). The Mindlin simplified theory in which strain energy is only a function of first-order gradient of strain tensor contains five new constants as well as Lame's constants for an isotropic linear elastic material (Mindlin, 1964; Askes and Aifantis, 2011). It can be shown that these five new constants can be grouped in two new constants (Askes and Aifantis, 2011). In this way, the number of new material constants is reduced from 5 down to 2 (Askes and Aifantis, 2011). A simplified kinetic energy whose constitutive equation consists of velocity and velocity gradient was also suggested by Mindlin (1964). Therefore, Mindlin's simplified theory contains one additional inertia parameter as well as two additional elastic parameters (Askes and Aifantis, 2011).

Lam et al. (2003) proposed a modified strain gradient theory in which three new length scale parameters were introduced into isotropic linear elastic materials. In this theory, the total deformation energy density is independent of the anti-symmetric rotation gradient tensor and it is only a function of the symmetric strain tensor, the dilatation gradient vector, the deviatoric stretch gradient tensor and the symmetric rotation gradient tensor (Lam et al., 2003).

The other well-known higher-order continuum theories are classical couple stress theory (Mindlin and Tiersten, 1962) with two material length scale parameters for an isotropic elastic material, and the modified couple stress theory (Yang et al., 2002) whose modified constitutive equation contains only a new length scale parameter. It can be shown that the modified couple stress theory is a special case of the modified strain gradient elasticity theory if two of the three material length scale parameters of the modified strain gradient theory are taken to be equal to zero (Ashoori Movassagh and Mahmoodi, 2013).

On the basis of Aifantis' studies in plasticity and nonlinear elasticity, Aifantis and his coworkers proposed another gradient elasticity theory, the constitutive equation of which is a function of strain and Laplacian of strain with one internal length (Aifantis, 1992; Altan and Aifantis, 1997). It can be shown that the associated equilibrium equations obtained by simplified Midlin's theory can be converted to those obtained by the Aifantis gradient elasticity theory if two new elastic parameters of simplified Mindlin's theory are taken to be equal to each other (Askes and Aifantis, 2011).

Another powerful gradient elasticity theory proposed to simulate dynamical behavior of micro/nano structures is achieved by combining stable strain gradients with acceleration (inertia) gradients (Askes and Aifantis, 2011) such as Mindlin's simplify theory. Metrikine and Askes 
(2002, 2006) derived such a theory from a discrete lattice. It is worth mentioning that these theories known as dynamically consistent models (Askes and Aifantis, 2011), incorporate at least two length scales: one of them related to strain gradients and another related to acceleration (inertia) gradients.

The nonlocal strain gradient theory is another Laplacian based gradient elasticity whose constitutive equation is a function of strain, stress, Laplacian of strain and Laplacian of stress (Askes and Aifantis, 2011; Aifantis, 2011).

Meany researchers employed different gradient elasticity theories mentioned above in conjunction with different classical continuum mechanic theories to study the mechanical behavior of size-dependent micro/nanostructures so far (Ansari et al., 2011, 2013; Reddy, 2011; Rahmani and Pedram, 2014; Akgöz and Civalek, 2012, 2014, 2015; Gholami et al., 2016; Binglei et al. 2011, 2016; Askes and Aifantis, 2009; Şimşek, 2016; Ebrahimi et al., 2016; Li and Hu, 2016).

On the basis of the strain gradient Timoshenko beam theory, the free vibration characteristics of functionally graded microbeams were investigated by Ansari et al. (2011). Ansari et al. (2013) also combined the most general strain gradient elasticity theory containing five additional material length scale parameters with the classical Timoshenko beam theory to investigate bending and buckling of functionally graded microbeams. Akgöz and Civalek (2014) proposed a new size-dependent trigonometric beam model based on the strain gradient theory. They (Akgöz and Civalek, 2014) employed their new model to estimate load-bearing capacity of microbeams. A new size-dependent sinusoidal plate model to predict mechanical behavior of thin, moderately thick and thick microplate was suggested by Akgöz and Civalek (2015) as well. They employed a modified strain gradient elasticity hypothesis to incorporate the size effect into the classical continuum plate theory. In an earlier work, Akgöz and Civalek (2012) estimated vibratory behavior of single-layered graphene sheets embedded in an elastic matrix. To this end, they used the Krichhoff plate hypothesis in conjunction with the modified couple stress theory to develop governing equations of motion (Akgöz and Civalek, 2012).

Gholami et al. (2016) combined Mindlin's simplified theory hypothesis with first-order shear deformation shell theory to investigate free vibration and axial buckling of circular cylindrical micro-/nano-shells. They ignored the effects of inertia gradients in their model and derived the kinetic energy in terms of macroscopic kinematic quantities. They compared three different sizedependent shell models on the basis of strain gradient theory, modified strain gradient theory, and modified couple stress theory in predicting the natural frequency and load-bearing capacity of microshells as well. They showed that the values of natural frequency and the critical buckling force predicted by the strain gradient theory are higher than those predicted by the modified strain gradient theory because of inducing a higher stiffness. Binglei et al. (2011) simulated static bending, static instability and vibratory behavior of a simply-supported micro-plate via the modified strain gradient theory and the modified couple stress theory. Their results clearly show that the strain gradient theory induces a higher stiffness than the modified couple stress theory. They (Binglei et al., 2016) re-derived the governing equation of the nonclassical Kirchhoff micro-plate as well as the general boundary conditions based on the strain gradient elasticity to simulate mechanical behavior of a micro-plate with any reasonable boundary conditions.

Askes and Aifantis (2009) employed a dynamically consistent model with two length scales to simulate flexural wave dispersion in carbon nanotubes. They verified validity of the proposed model by comparing their findings with those obtained via molecular dynamics simulation and/or Eringen's nonlocal elasticity theory.

Using nonlocal strain gradient elasticity, $\mathrm{Li}$ and $\mathrm{Hu}$ (2016) developed a model to analyze wave propagation in fluid-conveying carbon nanotubes. They investigated the effects of different parameters such as nonlocal parameter, small scale parameter, damping coefficient and flow velocity on wave propagation properties of fluid-conveying carbon nanotubes.

The other well-known gradient theory is Eringen's nonlocal elasticity theory in which there is 
only one length scale parameter and its constitutive equation is expressed based on the nonlocal stress tensor and Laplacian stress tensor (Askes and Aifantis, 2011). Eringen's nonlocal elasticity is widely employed to simulate mechanical behavior of carbon nano-structures because of being a good agreement between obtained results via Eringen's theory and molecular dynamics simulation. Murmu and Pradhana (2009) employed a nonlocal elasticity theory to investigate the vibrational behavior of SLGS. Numerical results clearly revealed that the small scale value could affect the fundamental frequencies of SLGS significantly (Murmu and Pradhana, 2009). Shen et al. (2010) employed a nonlocal orthotropic plate model which contained small scale effects to simulate nonlinear vibration behavior of SLGS in thermal environmental. They used temperature-dependent material properties obtained by molecular dynamic simulation. To estimate the value of the small-scale parameter, they reconciled the natural frequencies of graphene sheets obtained via molecular dynamics (MD) simulation with those predicted by the nonlocal plate model (Shen et al., 2010). Their results clearly showed the significant impact of vibration amplitude and temperature change on the nonlinear vibration response of both armchair and zigzag graphene sheets (Shen et al., 2010). Ansari et al. (2010) employed the nonclassical Mindlin plate theory to study the vibrational behavior of SLGS. To incorporate the size effect into the continuum plate model, they used Eringen's nonlocal elasticity theory. Matching the results obtained from MD simulation with those taken from the nonlocal model, they determined the proper value of the nonlocal parameter. They showed that the nonlocal parameter depends on chairality and boundary conditions. Using nonlocal elasticity, Farajpour et al. (2011) estimated the load-bearing capacity of circular graphene sheet subjected to uniform radial compression. To study the vibratory behavior of annular and circular graphene sheet in thermal environment, Mohammadi et al. (2014) employed Eringen's theory. They investigated the effect of different parameters such as elastic medium, boundary conditions and small scale parameter on the natural frequencies of circular and annular graphene sheets. Farajpour et al. (2012) estimated load-bearing capacity of a rectangular single-layered graphene sheet by combining the nonlocal elasticity theory with Kirchhoff's plate hypothesis as well as orthotropic material stress-strain relations.

Although there have been numerous studies carried out on the simulation of vibrational behavior of perfect graphene sheets, the notable studies shown that the effects of nanopores on linear and/or nonlinear vibration responses of graphene sheets do not exist, while the existence of nanopores in the graphene lattice can be essential based upon the desired functionality of graphene sheets in different nano-devices such as bio-devices and the DNA-decorated graphene (Zandiatashbar et al., 2014). On the other hand, the production process used or environmental and operating conditions under which the graphene device operates can lead to the appearance of vacancy defects in the graphene lattice (Zandiatashbar et al., 2014). Therefore, simulation of the dynamic response of graphene sheets with nanopores to determine how the size, the position and the shape of cut-outs influence the natural frequencies is important. Accordingly, the main purpose of this study is to model the dynamic response of SLGS with cut-outs. Different methods, such as Ritz, finite difference and finite element can be employed to this end. Rajamani and Prabhakaran (1977) used Lagrange's equations to obtain discrete equations of motion of a composite plate with a central cut-out. For this purpose, they employed potential energy and kinetic energy of a uniform plate and considered the cut-out as a displacement-dependent external loading on the plate (Rajamani and Prabhakaran, 1977). The finite difference method was used by Aksu and Ali (1976) to determine vibration characteristics of rectangular plates with one or two central cut-outs. To develop a simple method to study the effects of the rectangular cutout on natural frequencies of rectangular plates, Ali and Atwal (1980) used Rayleigh's method and employed trigonometric functions to estimate the lateral deflection. They showed that this method can predict the fundamental frequency with a quite good accuracy although the accuracy could be increased if one added a correction function to the estimated lateral displacement 
function (Ali and Atwal, 1980). Lam et al. (1989) presented a modification of the Rayleigh-Ritz method to investigate the vibrational behavior of rectangular plates with one or two cut-outs. They used an orthogonal polynomial function generated based on the Gram-Schmidt process to estimate the lateral displacement of plate segments (Lam et al., 1989). Similar methods were used by Liew et al. (2003) to investigate the effect of different boundary conditions on vibrational behavior of a rectangular plate with the central rectangular cut-out. The negative stiffness method was applied to analyze free vibration and buckling of plates with cut-outs by Tham et al. (1986).

One of the popular variational methods employed to derive discrete equations of motion is the Ritz method because the displacement field is approximated by a linear combination of shape functions which are only satisfied by essential boundary conditions. On the other hand, it is shown that one can reduce the complexity of the mechanical behavior modelling of structures with cut-outs by combining the construction of energy functional with the Ritz method (Malekzadeh et al., 2013). Therefore, in this study, the Ritz method is employed to derive discrete equations of motion which yield the eigenvalue problem. Because Eringen's nonlocal elasticity theory does not allow the construction of energy functional (Reddy, 2011), the dynamic consistent model is used to simulate vibrational behavior of the graphene sheet with a nanopore. To this end, Aifantis' theory, as well as inertia gradients is combined with Kirchhoff's thin plate hypothesis to construct the energy functional and incorporate the size effect into the classical continuum plate model. Also, the length scales related to strain gradients and inertia gradients are set equal to each other. The molecular structural mechanics method is also used to simulate vibrational behavior of defective SLGS to estimate the length scale parameter which must be used in the proposed nonclassical plate model.

\section{Governing equation}

\subsection{Nonclassical thin plate theory}

According to Hamilton's principle, one can formulate discretized free vibration equations of a nano-plate with free edges cut-out via the Ritz method. The Aifantis theory in conjunction with inertia gradients is also used to incorporate the size effect into the equation governing natural frequencies of the nano-plate with the cut-out. For this reason, the variation of strain energy and kinetic energy can be written as

$$
\begin{aligned}
\delta P E & =\int_{V_{I}}\left(\sigma_{i j} \delta \varepsilon_{i j}^{L}\right) d V-\int_{V_{I I}}\left(\sigma_{i j} \delta \varepsilon_{i j}^{L}\right) d V \\
\delta K E & =\int_{V_{I}}\left[\rho \dot{u} \delta \dot{u}+\rho \dot{v} \delta \dot{v}+\rho \dot{w} \delta \dot{w}+\rho l_{1}^{2}\left(\dot{u}_{, i} \delta \dot{u}_{, i}+\dot{v}_{, i} \delta \dot{v}_{, i}+\dot{w}_{, i} \delta \dot{w}_{, i}\right)\right] d V \\
& -\int_{V_{I I}}\left[\rho \dot{u} \delta \dot{u}+\rho \dot{v} \delta \dot{v}+\rho \dot{w} \delta \dot{w}+\rho l_{1}^{2}\left(\dot{u}_{, i} \delta \dot{u}_{, i}+\dot{v}_{, i} \delta \dot{v}_{, i}+\dot{w}_{, i} \delta \dot{w}_{, i}\right)\right] d V
\end{aligned}
$$

where $\sigma_{i j}, \varepsilon_{i j}^{L}$ and $l_{1}$ are components of the stress tensor, linear strain tensor and length scale related to inertia gradients, respectively. $V_{i}(i=I, I I)$ is the volume of $i$-th segment of the plate. The displacement field is shown by $u, v$ and $w . \rho$ is the specific mass density of the plate material and the time derivative of displacements are shown by overhead dot. According to the classical plate theory hypothesis, if the $x y$-plane of the Cartesian coordinate system $(x, y, z)$ coincides with the geometrical mid-plane of the undeformed rectangular micro-/nano-plate, the displacement field can be expressed as (Reddy, 1999) 


$$
\begin{aligned}
& u(x, y, z, t)=u_{0}(x, y, t)-z \frac{\partial w(x, y, t)}{\partial x} \quad v(x, y, z, t)=v_{0}(x, y, t)-z \frac{\partial w(x, y, t)}{\partial y} \\
& w(x, y, z, t)=w(x, y, t)
\end{aligned}
$$

where $u_{0}$ and $v_{0}$ are the $x$ - and $y$-components of the displacement vector of a point in the mid-plane of the plate at time $t$, respectively.

According to the classical plate theory hypothesis, the linear components of the strain tensor can be found on the basis of Eqs. (2.2) (Reddy, 1999)

$$
\begin{aligned}
& \varepsilon_{x x}^{L}=\frac{\partial u_{0}}{\partial x}-z \frac{\partial^{2} w}{\partial x^{2}} \quad \varepsilon_{y y}^{L}=\frac{\partial v_{0}}{\partial y}-z \frac{\partial^{2} w}{\partial y^{2}} \\
& \varepsilon_{x y}^{L}=\varepsilon_{y x}^{L}=\frac{1}{2}\left(\frac{\partial v_{0}}{\partial x}+\frac{\partial u_{0}}{\partial y}-2 z \frac{\partial^{2} w}{\partial x \partial y}\right)
\end{aligned}
$$

According to Aifantis' theory, the stress tensor components are related to the linear strain tensor components as (more details can be found in Aifantis (1992, 2011), Askes and Aifantis (2011), Gitman et al. (2005))

$$
\sigma_{i j}=C_{i j k l}\left(\varepsilon_{k l}-l^{2} \varepsilon_{k l, m m}\right)
$$

which can be obtained on the basis of the implicit gradient elasticity model given by

$$
\operatorname{tr}\left(\alpha_{1} \varepsilon+\alpha_{2} \boldsymbol{\sigma}\right) \mathbf{1}+\alpha_{3} \boldsymbol{\varepsilon}+\alpha_{4} \boldsymbol{\sigma}+\nabla^{2}\left[\operatorname{tr}\left(\alpha_{5} \boldsymbol{\varepsilon}+\alpha_{6} \boldsymbol{\sigma}\right) \mathbf{1}+\alpha_{7} \boldsymbol{\varepsilon}+\alpha_{8} \boldsymbol{\sigma}\right]=0
$$

through a proper choice of the constants $\alpha_{1}, \ldots, \alpha_{8}$ (Aifantis, 2011). C can be defined based on the classical plate theory hypothesis as

$$
\mathbf{C}=\left[\begin{array}{ccc}
\frac{E}{1-\nu^{2}} & \frac{E \nu}{1-\nu^{2}} & 0 \\
\frac{E \nu}{1-\nu^{2}} & \frac{E}{1-\nu^{2}} & 0 \\
0 & 0 & G
\end{array}\right]
$$

where $E, G$ and $\nu$ are the modulus of elasticity, modulus of rigidity and Poisson's ratio, respectively. $l$ is the length scale parameter related to strain gradients.

By substituting Eqs. (2.2) to (2.5) into Eqs. (2.1), one can find the variation of strain energy and kinetic energy on the basis of the displacement field. By using the appropriate approximation of dependent unknowns $\left(u_{0}, v_{0}, w\right)$ satisfying the essential boundary conditions and employing Hamilton's principle, one can find the following system of ordinary differential equations governing the lateral vibration of the plate. It is completely independent of the other two equations governing in-plane motion of the plate

$$
\left(M_{m n p q}^{I}-M_{m n p q}^{I I}\right) \ddot{W}_{p q}+\left(K_{m n p q}^{I}-K_{m n p q}^{I I}\right) W_{p q}=0
$$

in which

$$
\begin{aligned}
& M_{m n p q}^{I, I I}=\int_{V_{I}, V_{I I}}\left[\frac{-1}{12} \rho h^{3}\left(\frac{\partial \phi_{p}}{\partial x} \frac{\partial \phi_{m}}{\partial x} \varphi_{q} \varphi_{n}+\frac{\partial \varphi_{q}}{\partial y} \frac{\partial \varphi_{n}}{\partial y} \phi_{p} \phi_{m}\right)-\rho h \phi_{p} \varphi_{q} \phi_{m} \varphi_{n}\right] d x d y \\
& -l_{1}^{2} \int_{V_{I}, V_{I I}} 2 \rho h\left(\frac{\partial \phi_{p}}{\partial x} \frac{\partial \phi_{m}}{\partial x} \varphi_{q} \varphi_{n}+\frac{\partial \varphi_{q}}{\partial y} \frac{\partial \varphi_{n}}{\partial y} \phi_{p} \phi_{m}\right) d x d y \\
& -l_{1}^{2} \int_{V_{I}, V_{I I}} \frac{1}{12} \rho h^{3}\left(\frac{\partial^{2} \phi_{p}}{\partial x^{2}} \frac{\partial^{2} \phi_{m}}{\partial x^{2}} \varphi_{q} \varphi_{n}+\frac{\partial^{2} \varphi_{q}}{\partial y^{2}} \frac{\partial^{2} \varphi_{n}}{\partial y^{2}} \phi_{p} \phi_{m}+2 \frac{\partial \phi_{p}}{\partial x} \frac{\partial \phi_{m}}{\partial x} \frac{\partial \varphi_{q}}{\partial y} \frac{\partial \varphi_{n}}{\partial y}\right) d x d y
\end{aligned}
$$


and

$$
\begin{aligned}
& K_{m n p q}^{I, I I}=\int_{V_{I}, V_{I I}}\left[-D\left(\frac{\partial^{2} \phi_{p}}{\partial x^{2}} \varphi_{q}+\nu \frac{\partial^{2} \varphi_{q}}{\partial y^{2}} \phi_{p}\right) \frac{\partial^{2} \phi_{m}}{\partial x^{2}} \varphi_{n}\right. \\
& \left.-D\left(\frac{\partial^{2} \varphi_{q}}{\partial y^{2}} \phi_{p}+\nu \frac{\partial^{2} \phi_{p}}{\partial x^{2}} \varphi_{q}\right) \frac{\partial^{2} \varphi_{n}}{\partial y^{2}} \phi_{m}-[2 D(1-\nu)] \frac{\partial^{2}\left(\phi_{p} \varphi_{q}\right)}{\partial x \partial y} \frac{\partial^{2}\left(\phi_{m} \varphi_{n}\right)}{\partial x \partial y}\right] d x d y \\
& -l^{2} \int_{V_{I}, V_{I I}}\left[-D\left(\frac{\partial^{4} \phi_{p}}{\partial x^{4}} \varphi_{q}+(1+\nu) \frac{\partial^{2} \varphi_{q}}{\partial y^{2}} \frac{\partial^{2} \phi_{p}}{\partial x^{2}}+\nu \frac{\partial^{4} \varphi_{q}}{\partial y^{4}} \phi_{p}\right) \frac{\partial^{2} \phi_{m}}{\partial x^{2}} \varphi_{n}\right. \\
& -D\left(\frac{\partial^{4} \varphi_{q}}{\partial y^{4}} \phi_{p}+(1+\nu) \frac{\partial^{2} \phi_{p}}{\partial x^{2}} \frac{\partial^{2} \varphi_{q}}{\partial y^{2}}+\nu \frac{\partial^{4} \phi_{p}}{\partial x^{4}} \varphi_{q}\right) \frac{\partial^{2} \varphi_{n}}{\partial y^{2}} \phi_{m} \\
& \left.-[2 D(1-\nu)]\left(\frac{\partial^{4}\left(\phi_{p} \varphi_{q}\right)}{\partial x^{3} \partial y}+\frac{\partial^{4}\left(\phi_{p} \varphi_{q}\right)}{\partial x \partial y^{3}}\right) \frac{\partial^{2}\left(\phi_{m} \varphi_{n}\right)}{\partial x \partial y}\right] d x d y
\end{aligned}
$$

where $D=E h^{3} /\left[12\left(1-\nu^{2}\right)\right]$ and $h$ is thickness of the plate. The Gram-Schmidt process is used to generate the polynomial functions $\varphi_{n}(y)\left(\varphi_{q}(y)\right)$ and $\phi_{m}(x)\left(\phi_{p}(x)\right)$ as well.

Equation (2.6) can be solved on the basis of the eigen-value problem to find the natural frequencies corresponding to lateral vibration of the nano-plate.

\subsection{Molecular structural mechanics}

The molecular structural mechanics approach has been built on the basis of some similarities between the molecular model of carbon nanostructures (nanotube and graphene sheet) and the structure of a space frame building ( $\mathrm{Li}$ and Chou, 2003; Hu et al., 2007; Sakhaee-pour et al., 2008; Hashemnia et al., 2009; Gupta and Batra, 2010, Sadeghi and Naghdabadi, 2010; Wang et al., 2013). In this method, the atomic lattice of carbon nanostructures is rebuilt by beams and point masses replacing C-C covalent bonds and carbon atoms, respectively ( $\mathrm{Li}$ and Chou, 2003; Hu et al., 2007; Sakhaee-pour et al., 2008; Hashemnia et al., 2009; Gupta and Batra, 2010; Sadeghi and Naghdabadi, 2010; Wang et al., 2013). To determine stiffness parameters of the equivalent beam, i.e., tensile stiffness (EA), bending stiffness (EI) and torsional rigidity (GJ), one can use the concepts of energy equivalence between the total potential energies in computational chemistry (Eq. (2.9)) and the total elemental strain energies in structural mechanics (Eq. (2.10)) (Li and Chou, 2003; Hu et al., 2007; Sakhaee-pour et al., 2008; Hashemnia et al., 2009; Gupta and Batra, 2010; Sadeghi and Naghdabadi, 2010; Wang et al., 2013).

The total potential function among carbon atoms due to bonded and nonbonded interactions are (Li and Chou, 2003; Hu et al., 2007; Sakhaee-pour et al., 2008; Hashemnia et al., 2009; Gupta and Batra, 2010; Sadeghi and Naghdabadi, 2010; Wang et al., 2013)

$$
U_{P}=\sum U_{r}+\sum U_{\theta}+\sum\left(U_{\varphi}+U_{\omega}\right)+\sum U_{v d w}
$$

in which $U_{r}$ is the bond stretching, $U_{\theta}$ is the bond angle bending, $U_{\varphi}$ is the dihedral angle torsion, $U_{\omega}$ is the out of plane torsion, $U_{v d w}$ is the nonbonded van der Waals interaction and $U_{e}$ is the nonbonded electrostatic interactions. On the other hand, the total strain energy for a beam element is ( $\mathrm{Li}$ and $\mathrm{Chou}, 2003$; Hu et al., 2007; Sakhaee-pour et al., 2008; Hashemnia et al., 2009; Gupta and Batra, 2010; Sadeghi and Naghdabadi, 2010; Wang et al., 2013)

$$
U_{S E}=\sum U_{A}+\sum U_{M}+\sum U_{T}+\sum U_{V}
$$

where $U_{A}$ is the strain energy of axial tension, $U_{M}$ is the strain energy of bending, $U_{T}$ is the strain energy of torsion and $U_{v}$ is the strain energy of the shear force. One can obtain a relationship between the molecular mechanics force field constants and the structural mechanics parameters 
by using the energy equivalence between Eq. (2.9) and Eq. (2.10) (Li and Chou, 2003; Hu et al., 2007; Sakhaee-pour et al., 2008; Hashemnia et al., 2009; Gupta and Batra, 2010; Sadeghi and Naghdabadi, 2010; Wang et al., 2013) as follows

$$
\frac{E A}{L}=k_{r} \quad \frac{E I}{L}=k_{\theta} \quad \frac{G J}{L}=k_{\tau}
$$

where $k_{r}, k_{\theta}$ and $k_{\tau}$ are force field constants in molecular mechanics.

On the basis of the previous research ( $\mathrm{Li}$ and $\mathrm{Chou}, 2003$; Hu et al., 2007; Sakhaee-pour et al., 2008; Hashemnia et al., 2009; Gupta and Batra, 2010; Sadeghi and Naghdabadi, 2010; Wang et al., 2013), the following numerical values of the force field constants used in this research are selected

$$
k_{r}=65.2 \mathrm{nNA}^{-1} \quad k_{\theta}=2.78 \mathrm{nNÅrad}^{-2} \quad k_{\tau}=2.78 \mathrm{nNÅrad}^{-2}
$$

Then, the diameter-to-length ratio of the beam element estimated by Eq. (2.11) shows that this element is too thick and must be modeled based on shear deformation beam theories.

Wang et al. (2013) used the study of Scarpa et al. (2009) who considered the C-C bond as a Timoshenko beam, to obtain the mechanical properties and optimized cross-section diameter of the equivalent beam element via the following relationships (Wang et al., 2013)

$$
d<2 \sqrt{6 \frac{k_{\theta}}{k_{r}}} \quad E=\frac{k_{r}^{2} L}{4 \pi k_{\theta}} \quad G=\frac{k_{\tau}}{2 k_{\theta}}
$$

where $k_{\theta}$ is expressed as

$$
k_{\theta}=\frac{k_{r} d^{2}}{16} \frac{4 C_{1}+C_{2}}{C_{1}+C_{2}}
$$

and (Wang et al., 2013)

$$
C_{1}=112 L^{2} k_{\tau}+192 L^{2} k_{\tau} \nu+64 L^{2} k_{\tau} \nu^{2} \quad C_{2}=9 k_{r} d^{4}+18 k_{r} d^{4} \nu+9 k_{r} d^{4} v^{2}
$$

and $L$ is length of the $\mathrm{C}-\mathrm{C}$ bond. They obtained the diameter-to-length ratio of the equivalent Timoshenko beam around 0.704 (Wang et al., 2013), which is used in this study.

Based on the finite element method and using modal analysis concepts, one can find the natural frequencies of this space frame-like structure constructed by Timoshenko beam elements and point masses.

\section{Verification}

\subsection{Non-classical thin plate theory}

Based on the best knowledge of the author, it is the first attempt to analyze the vibrational behavior of graphene sheets with nanopores via Aifantis' theory combined with acceleration (inertia) gradients and the Ritz method. Then, to verify the correctness of the presented model, the author first compares the obtained natural frequencies of classical thin plates with central holes with those obtained based on the finite element method (Table 1). To this end, the value of length scale parameters $\left(l\right.$ and $\left.l_{1}\right)$ is taken equal to zero.

According to Table 1, although natural frequencies of perfect plates can be accurately predicted by the present model, those of the defective plate are overestimated. However, the calculated percentage error (lower than $4 \%$ and $3 \%$ for the simply supported and clamped plate, respectively) shows that there is an acceptable agreement between the results. 
Table 1. Comparisons of the first dimensionless natural frequencies $\Omega=\left(\omega a^{2} / \pi^{2}\right) \sqrt{\rho h / D}$ for the fully simply supported (SSSS) and fully clamped (CCCC) plate $(a / b=1, a / h=29.4, d$ - the diameter of the central hole)

\begin{tabular}{|c|c|c|c|c|}
\hline \multirow{2}{*}{$d / a$} & \multicolumn{2}{|c|}{ SSSS } & \multicolumn{2}{c|}{ CCCC } \\
\cline { 2 - 5 } & Present & FEM & Present & FEM \\
\hline \hline 0 & 1.9986 & 1.9986 & 3.6445 & 3.6445 \\
\hline 0.1 & 2.0021 & 1.9913 & 3.6907 & 3.6533 \\
\hline 0.2 & 2.0304 & 1.9950 & 3.8482 & 3.7644 \\
\hline 0.3 & 2.0918 & 2.0454 & 4.1673 & 4.0898 \\
\hline 0.4 & 2.1979 & 2.1727 & 4.7690 & 4.7286 \\
\hline 0.5 & 2.4988 & 2.4106 & 5.8615 & 5.8571 \\
\hline
\end{tabular}

Next, after setting the length scale parameters to be equal to each other (i.e. $l=l_{1}$ ), the effects of an increase in the length scale parameter on the variation of the first natural frequency of perfect graphene sheets by increasing the side-length of nano-plate is compared with the data available in Ansari et al. (2010), showing the influence of the small scale parameter on the natural frequency of perfect graphene sheets by using Eringen's nonlocal elasticity theory. Figure 1 shows that, regardless of the boundary conditions, the first natural frequency of perfect graphene sheets decreases with an increase in the length scale parameter, although a rise in the side-length of the nano-plate reduces the importance of the length scale value, so that the non-classical natural frequencies tend to the classical ones. The observed behavior is completely in agreement with that reported in Ansari et al. (2010).
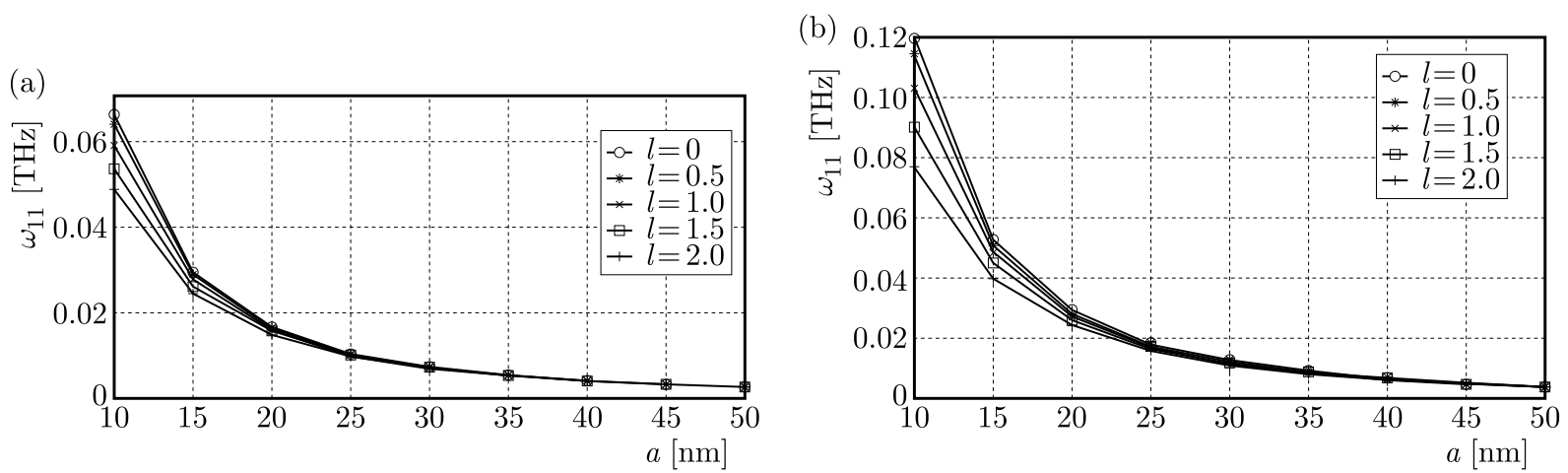

Fig. 1. The effects of the length scale value on the natural frequency of perfect graphene sheets; (a) fully simply supported nano-plates, (b) fully clamped nano-plates $\left(l=l_{1}\right)$

\subsection{Molecular structural mechanics method}

To verify the accuracy of predicted natural frequencies of graphene sheets obtained via molecular structural mechanics (MSM), the estimated first natural frequency of fully simply supported and fully clamped single-layered graphene sheets are compared with those obtained by the molecular dynamics method and are available in Ansari et al. (2010), see Table 2. As it is seen, there is a quite good agreement between the results.

\section{Numerical results}

In this Section, first, the effects of different parameters such as length scale and diameter of the central hole to the side length ratio, which is not more than the half of side length of nanoplate, on the dimensionless natural frequency of graphene nano-sheet with a central pore are 
Table 2. Comparison of the first natural frequency of a square single-layered graphene sheet

\begin{tabular}{|c|c|c|c|c|}
\hline Side-length & \multicolumn{2}{|c|}{ SSSS } & \multicolumn{2}{c|}{ CCCC } \\
\cline { 2 - 5 }$a[\mathrm{~nm}]$ & MSM & MD & MSM & MD \\
\hline \hline 10 & 0.0576347 & 0.0587725 & 0.1148990 & 0.1146223 \\
\hline 15 & 0.0292556 & 0.0273881 & 0.0523147 & 0.0517078 \\
\hline 20 & 0.0167249 & 0.0157524 & 0.0310192 & 0.0306219 \\
\hline 25 & 0.0118420 & 0.0099840 & 0.0182260 & 0.0179975 \\
\hline
\end{tabular}

investigated (see Fig. 2). Then, the variation of dimensionless natural frequencies of the graphene nano-sheet with the pore diameter to side length ratio equal to 0.5 versus the length scale and side length is studied. Finally, it is tried to estimate the effective length scale of the defective graphene sheet via matching the results obtained from the non-classical plate theory with those found on the basis of molecular structural mechanics. It should be mentioned that the length scales related to strain gradients and inertia gradients are set equal to each other (i.e. $l=l_{1}$ ) in this Section.

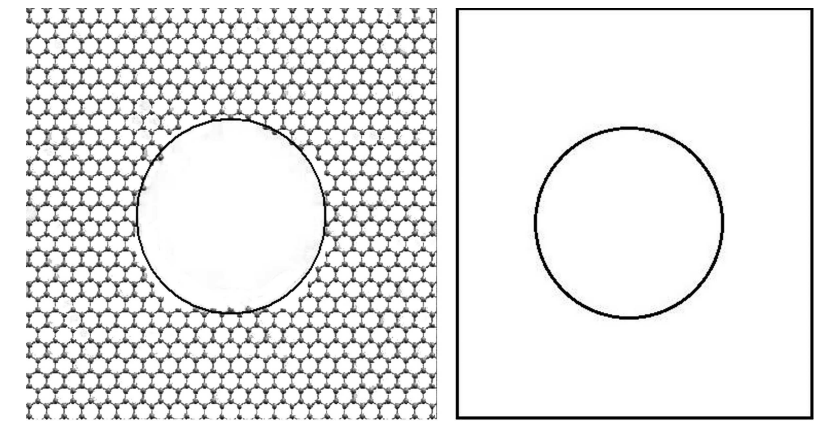

Fig. 2. Defective monolayer graphene sheet modeled with a plate with a circular hole

In Fig. 3, the effects of the length scale and diameter of the central hole to the side length ratio on the dimensionless natural frequency of the graphene nano-sheet with a central pore for two different boundary conditions are investigated. Regardless of boundary conditions, for a small value of the length scale parameter, the first natural frequency of defective graphene sheets rises as diameter of the central hole to the side length ratio increases, while with a rise in the length scale, the rate of increase of the first natural frequency with pore diameter decreases. For a larger value of the length scale, a decrease in the first natural frequency may be seen by increasing the pore diameter (Figs. 3a and 3d). Generally, however, the sensitivity of simply supported graphene sheets to the existence of the central hole is less than in the fully clamped nano-sheets. The sensitivity of the second and third natural frequency of a fully clamped graphene sheet to an increase in pore diameter is less than the first one, although the length scale value can change the increasing or decreasing trend of the curves.

The impact of side length on frequencies can be seen in Fig. 4 for fully clamped and simply supported graphene sheets. It is assumed that graphene has a central hole the diameter of which is half of the side length of the sheet.

It is clearly seen that the importance of the length scale role in decreasing the natural frequencies reduces with an increase in the side length of the defective sheet. It can be concluded that the influence of the length scale on higher order frequencies is more than the first natural frequency as well. As expected, the natural frequencies of simply-supported sheets are less than the fully clamped ones.

In order to show if the existence of vacancy defects makes a difference to the effective length scale used in the non-classical plate theory, the results obtained from the molecular structural 
(a)

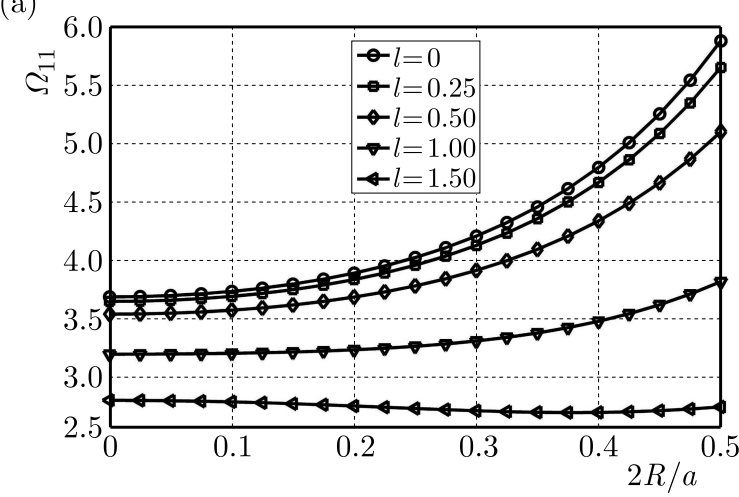

(b)

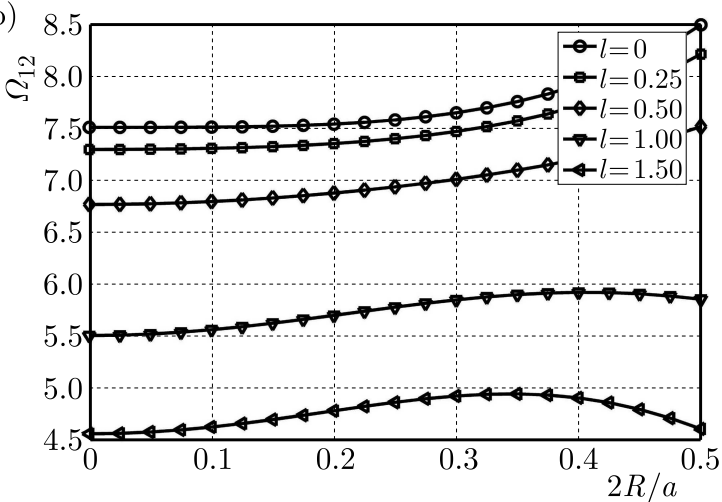

(c)

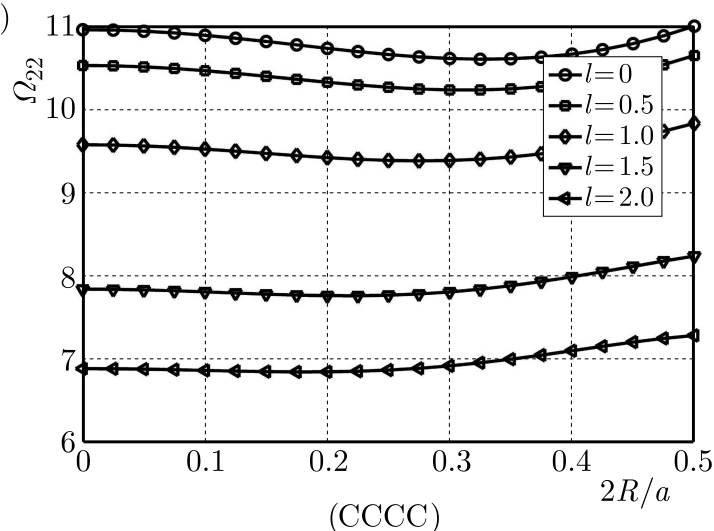

(d)

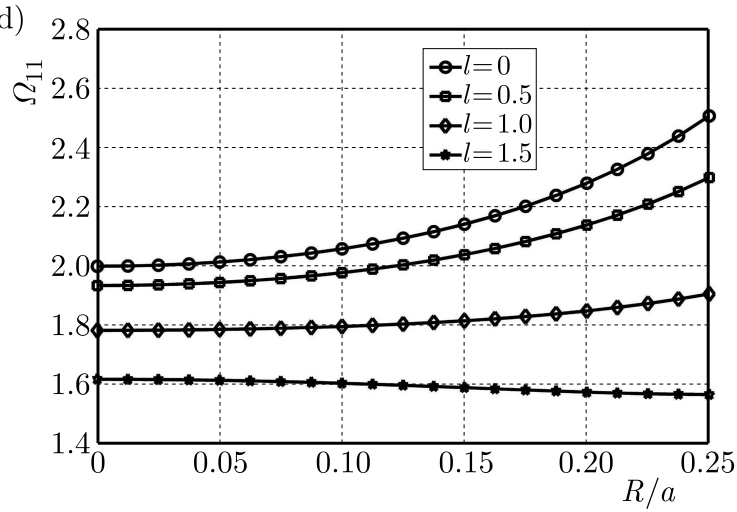

(e)

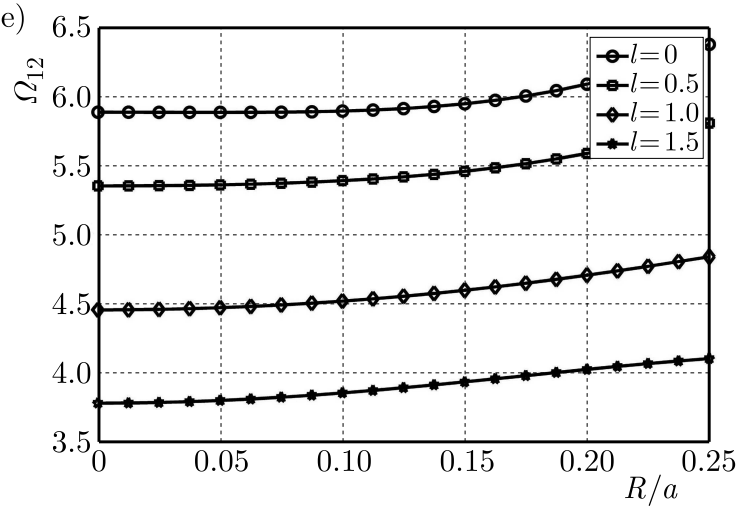

(f)

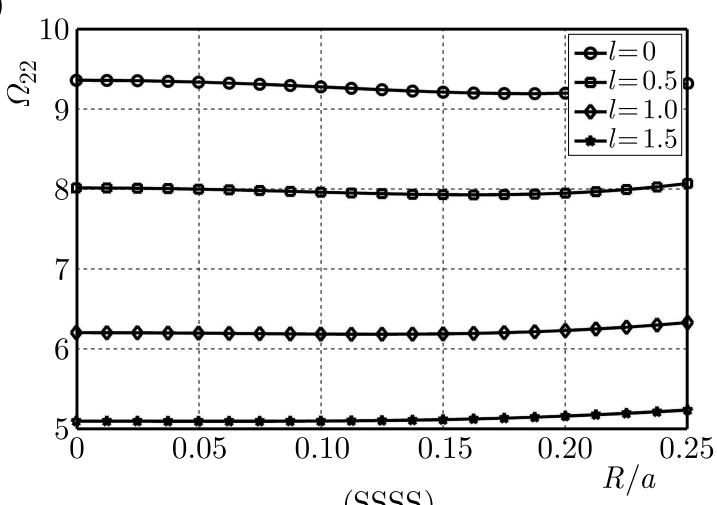

Fig. 3. The effects of the length scale and diameter of the hole to side length of plate ratio on the first three dimensionless natural frequencies of fully clamped graphene sheets (a), (b) and (c) and fully simply-supported graphene sheets (d), (e) and (f) $\left(l=l_{1}\right)$

mechanics are used. First, the effective length scale for perfect square graphene nano-sheets the side length of which is $10 \mathrm{~nm}$ is obtained by matching the first natural frequencies predicted by the molecular structural mechanics with the non-classical plate model. They are around $1.15 \mathrm{~nm}$ and $0.5 \mathrm{~nm}$ for simply supported and fully clamped nano-sheets, respectively. According to these effective length scales, the first three natural frequencies of nano-sheets are 0.0578181 , 0.133181 and $0.193658 \mathrm{THz}, 0.1150115,0.2230178$ and $0.3152479 \mathrm{THz}$ for simply supported and fully clamped nano-plates, respectively. The comparison of these results with those determined by molecular structural mechanics $(0.05763,0.12857$ and $0.18252 \mathrm{THz}$ for simply supported sheet and $0.11490,0.23047$ and 0.34411 for clamped sheet) shows that the percentage error in higher order frequencies is more than that of the first natural frequencies. Similar results can be seen in Table 3 and 4 in which the same method is used to estimate the effective length scale of the defective sheet. It can be concluded that the effective length scale may vary with an increase 
(a)

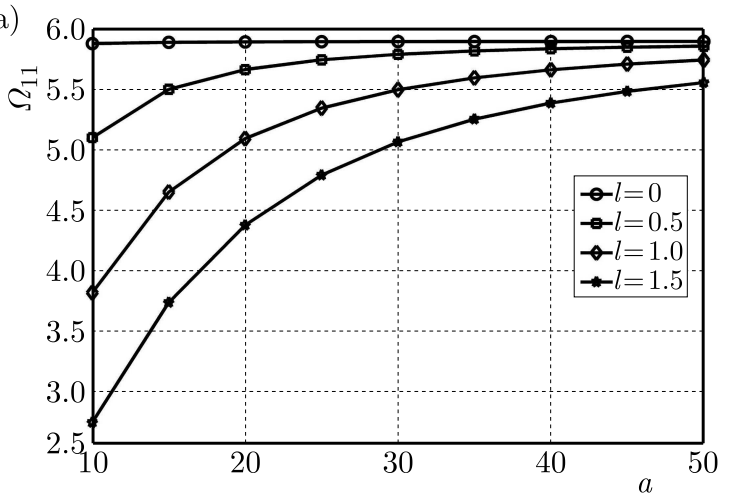

(b)

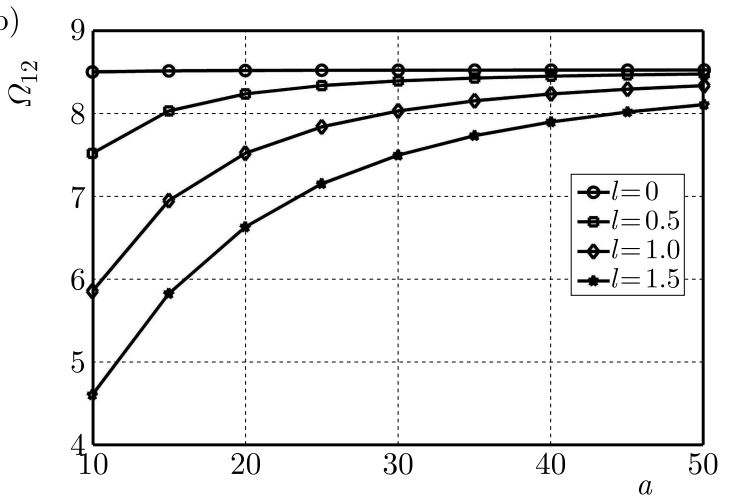

(c)

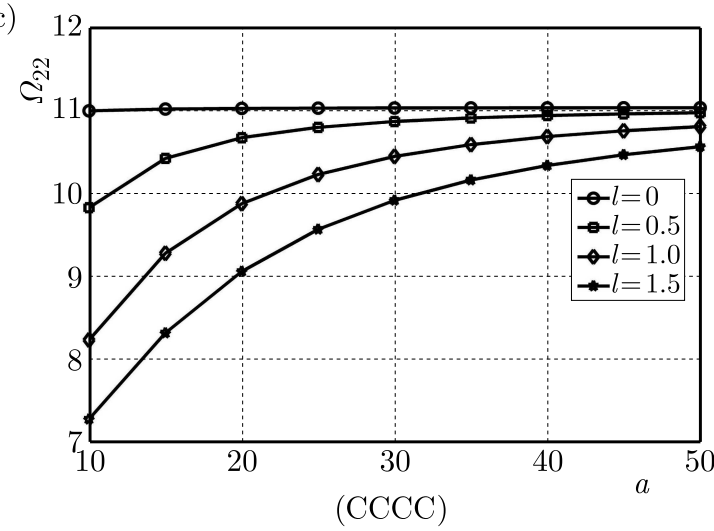

(d)

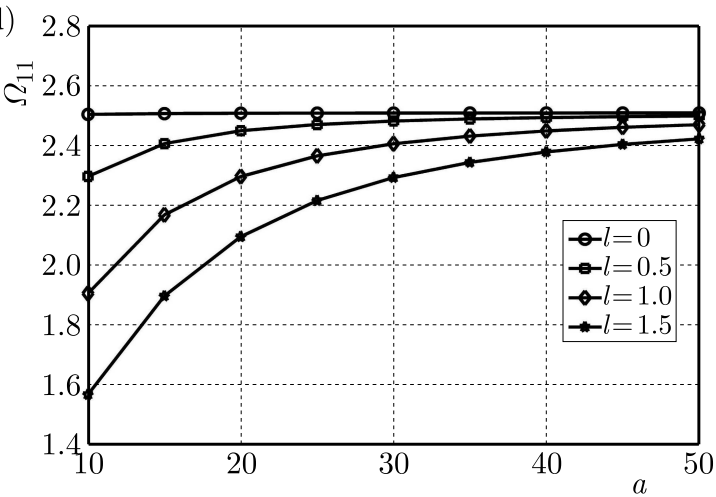

(e)

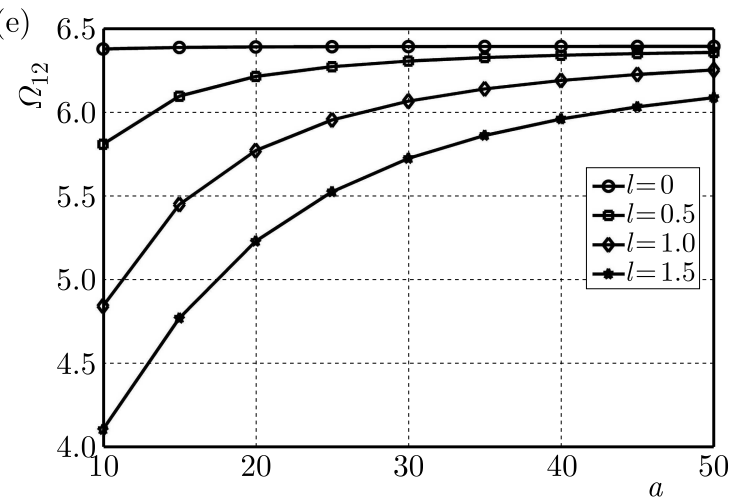

(f)

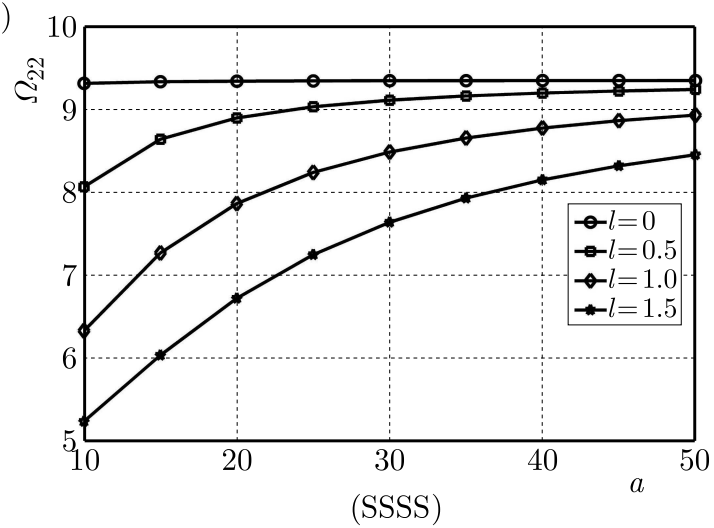

Fig. 4. The effect of the length scale and side length on the first three dimensionless natural frequencies of the fully clamped graphene sheet (a), (b) and (c) and fully simply-supported graphene sheet (d), (e) and (f) with a central hole the diameter to side length ratio of which is $0.5\left(l=l_{1}\right)$

in diameter of the vacancy defect as well. It seems that the dependency of the effective length scale on diameter of the vacancy defect changes with boundary conditions. The effective length scale of a clamped defective graphene is more sensitive to diameter of the vacancy than that of the simply supported one.

\section{Conclusion}

This article attempts to study the natural frequency of defective graphene sheets because the existence of cut-outs in plates may be essential on the basis of their desired functionality. In this study, Aifantis' theory in conjunction with inertia gradients is combined with Kirchhoff's thin plate hypothesis to incorporate the size effect into the classical continuum plate theory because Eringen's nonlocal elasticity theory does not allow the construction of an energy functional. 
Table 3. The estimated length-scale parameter of simply supported defective graphene sheets on the basis of matching natural frequencies obtained from the present method against MSM results

\begin{tabular}{|c|c|c|c|c|}
\hline $\begin{array}{c}\text { No. of } \\
\text { lost } \\
\text { atoms }\end{array}$ & $\begin{array}{c}\text { Mode } \\
\text { No. }\end{array}$ & $\begin{array}{c}\text { MSM } \\
{[\mathrm{THz}]}\end{array}$ & $\begin{array}{c}\text { Present } \\
\text { method } \\
{[\mathrm{THz}]}\end{array}$ & $\begin{array}{c}\text { Length scale } \\
\text { parameter } \\
l=l_{1}[\mathrm{~nm}]\end{array}$ \\
\hline \hline \multirow{3}{*}{6} & $\omega_{11}$ & 0.057446 & 0.05738 & \multirow{2}{*}{1.2} \\
\cline { 2 - 4 } & $\omega_{12}$ & 0.12840 & 0.14056 & \multirow{2}{*}{1.2} \\
\cline { 2 - 4 } & $\omega_{22}$ & 0.18224 & 0.19349 & \\
\hline \multirow{3}{*}{24} & $\omega_{11}$ & 0.057170 & 0.05711 & \multirow{2}{*}{1.145} \\
\cline { 2 - 4 } & $\omega_{12}$ & 0.12797 & 0.13944 & \multirow{2}{*}{1.155} \\
\cline { 2 - 4 } & $\omega_{22}$ & 0.18157 & 0.19140 & \\
\hline \multirow{3}{*}{54} & $\omega_{11}$ & 0.057008 & 0.05706 & \multirow{2}{*}{} \\
\cline { 2 - 4 } & $\omega_{12}$ & 0.12718 & 0.13934 & \multirow{2}{*}{1.155} \\
\cline { 2 - 4 } & $\omega_{22}$ & 0.18049 & 0.19096 & \\
\hline
\end{tabular}

Table 4. The estimated length-scale parameter of fully clamped defective graphene sheets on the basis of matching natural frequencies obtained from the present method against MSM results

\begin{tabular}{|c|c|c|c|c|}
\hline $\begin{array}{c}\text { No. of } \\
\text { lost } \\
\text { atoms }\end{array}$ & $\begin{array}{c}\text { Mode } \\
\text { No. }\end{array}$ & $\begin{array}{c}\text { MSM } \\
{[\mathrm{THz}]}\end{array}$ & $\begin{array}{c}\text { Present } \\
\text { method } \\
{[\mathrm{THz}]}\end{array}$ & $\begin{array}{c}\text { Length scale } \\
\text { parameter } \\
l=l_{1}[\mathrm{~nm}]\end{array}$ \\
\hline \hline \multirow{3}{*}{6} & $\omega_{11}$ & 0.11444 & 0.11439 & \multirow{2}{*}{0.55} \\
\cline { 2 - 4 } & $\omega_{12}$ & 0.23712 & 0.21931 & \multirow{2}{*}{0.55} \\
\cline { 2 - 4 } & $\omega_{13}$ & 0.34343 & 0.30796 & \multirow{2}{*}{0.61} \\
\hline \multirow{3}{*}{24} & $\omega_{11}$ & 0.11381 & 0.11383 & \multirow{2}{*}{0.71} \\
\cline { 2 - 4 } & $\omega_{12}$ & 0.23650 & 0.21525 & \multirow{2}{*}{0.71350} \\
\cline { 2 - 4 } & $\omega_{13}$ & 0.34196 & 0.29900 & \\
\hline \multirow{3}{*}{54} & $\omega_{11}$ & 0.11356 & 0.11355 & \multirow{2}{*}{} \\
\cline { 2 - 4 } & $\omega_{12}$ & 0.23650 & 0.21236 & \\
\cline { 2 - 4 } & $\omega_{13}$ & 0.34196 & 0.29157 & \multirow{2}{*}{} \\
\hline
\end{tabular}

The Ritz method is employed to derive discrete equations of motion which yield the eigenvalue problem. The molecular structural mechanics method is also employed to specify the effective length scale parameter. In the 'numerical results' Section, the effects of different boundary conditions, length scale, diameter of hole to side length ratio and side length of nano-sheets on the fundamental frequency of graphene sheets are studied. The results demonstrate that:

- Regardless of boundary conditions, for a small value of the length scale parameter, the first natural frequency of defective graphene sheets rises as diameter of the central hole to the side length ratio increases, while with a rise in the length scale, the rate of increase of the first natural frequency with pore diameter decreases.

- For larger values of the length scale, by increasing pore diameter, a decrease in the first natural frequency may be seen.

- The sensitivity of simply supported graphene sheets to the existence of the central hole is less than that of the fully clamped nano-sheets.

- The importance of the length scale role in decreasing the natural frequencies reduces with an increase in the side length of the defective sheet. 
- The influence of the length scale on higher order frequencies is stronger than that on the first natural frequencies.

- Although the dependency of the effective length scale on diameter of the vacancy defect changes with boundary conditions, the effective length scale does not vary with an increase in diameter of the vacancy defect significantly.

\section{Acknowledgements}

The author gratefully acknowledges the support of Yasouj University under Grant No. Gryu-89111109.

\section{References}

1. Aifantis E.C., 1992, On the role of gradients in the localization of deformation and fracture, International Journal of Engineering Science, 30, 1279-1299

2. Aifantis E.C., 2011, On the gradient approach-relation to Eringen's nonlocal theory, International Journal of Engineering Science, 49, 1367-1377

3. AkgÖz B., Civalek Ö., 2012, Free vibration analysis for single-layered graphene sheets in an elastic matrix via modified couple stress theory, Materials and Design, 42, 167-171

4. AkgÖz B., CIVAleK Ö., 2014, A new trigonometric beam model for buckling of strain gradient microbeams, International Journal of Mechanical Sciences, 81, 88-94

5. Akgöz B., Civalek Ö., 2015, A microstructure-dependent sinusoidal plate model based on the strain gradient elasticity theory, Acta Mechanica, 226, 2277-2294

6. Aksu G., Ali R., 1976, Determination of dynamic characteristics of rectangular plates with cutouts using a finite difference formulation, Journal of Sound and Vibration, 44, 147-158

7. Ali R., Atwal S.J., 1980, Prediction of natural frequencies of vibration of rectangular plates with rectangular cutouts, Composite Structures, 12, 819-823

8. Altan B., Aifantis E.C., 1997, On some aspects in the special theory of gradient elasticity, Journal of Mechanical Behavior of Material, 8, 231-282

9. Ansari R., Gholami R., Faghin Shojaei M., Mohammadi V., Sahmani S., 2013, Size-dependent bending, buckling and free vibration of functionally graded Timoshenko microbeams based on the most general strain gradient theory, Composite Structures, 100, 385-397

10. Ansari R., Gholami R., Sahmani S., 2011, Free vibration analysis of size-dependent functionally graded microbeams based on the strain gradient Timoshenko beam theory, Composite Structures, 94, 221-228

11. Ansari R., Sahmani S., Arash B., 2010, Nonlocal plate model for free vibration of single-layered graphene sheets, Physics Letters A, 375, 53-62

12. Ashoori Movassagh A., Mahmoodi A.M.J., 2013, A micro-scale modeling of Kirchhoff plate based on modified strain-gradient elasticity theory, European Journal of Mechanics A/Solids, 40, $50-59$

13. Askes H., Aifantis E.C., 2009, Gradient elasticity and flexural wave dispersion in carbon nanotubes, Physical Review, B80, 195412

14. Askes H., Aifantis E.C., 2011, Gradient elasticity in statics and dynamics: An overview of formulations, length scale identification procedures, finite element implementations and new results, International Journal of Solids and Structures, 48, 1962-1990

15. Binglei W., Shenghua H., Junfeng Z., Shenjie Z., 2016, Reconsiderations on boundary conditions of Kirchhoff micro-plate model based on a strain gradient elasticity theory, Applied Mathematical Modelling, 40, 7303-7317 
16. Binglei W., Shenjie Z., Junfeng Z., Xi C., 2011, A size-dependent Kirchhoff micro-plate model based on strain gradient elasticity theory, European Journal of Mechanics A/Solids, 30, $517-524$

17. Ebrahimi F., Barati M.R., Dabbagh A., 2016, A nonlocal strain gradient theory for wave propagation analysis in temperature-dependent inhomogeneous nanoplates, International Journal of Engineering Science, 107, 169-182

18. Farajpour A., Mohammadi M., Shahidi A.R., Mahzoon M., 2011, Axisymmetric buckling of the circular graphene sheets with the nonlocal continuum plate model, Physica E, 43, 1820-1825

19. Farajpour A., Shahidi A.R., Mohammadi M., Mahzoon M., 2012, Buckling of orthotropic micro/nanoscale plates under linearly varying in-plane load via nonlocal continuum mechanics, Composite Structures, 94, 1605-1615

20. Gholami R., Darvizeh A., Ansari R., Sadeghi F., 2016, Vibration and buckling of first-order shear deformable circular cylindrical micro-/nano-shells based on Mindlin's strain gradient elasticity theory, European Journal of Mechanics A/Solids, 58, 76-88

21. Gitman I.M., Askes H., Aifantis E.C., 2005, The representative volume size in static and dynamic micro-macro transitions, International Journal of Fracture, 135, L3-L9

22. Gupta S.S., Batra R.C., 2010, Elastic properties and frequencies of free vibrations of single-layered graphene sheets, Journal of Computational and Theoretical Nanoscience, 10, 2151-2164

23. Hashemnia K., FArid M., Vatankhah R., 2009, Vibrational analysis of carbon nanotubes and graphene sheets using molecular structural mechanics approach, Computational Materials Science, 47, 79-85

24. Hu N., Nunoya K., Pan D., Okabe T., Fukunaga H., 2007, Prediction of buckling characteristics of carbon nanotubes, International Journal of Solids and Structures, 44, 6535-6550

25. Jomehzadeh E., Saidi A.R., Jomehzadeh Z., Bonaccorso F., Palermo V., Galiotis C., Pugno N.M., 2015, Nonlinear subharmonic oscillation of orthotropic graphene-matrix composite, Computational Materials Science, 99, 164-172

26. Lam D.C.C., Yang F., Chong A.C.M., Wang J., Tong P., 2003, Experiments and theory in strain gradient elasticity, Journal of the Mechanics and Physics of Solids, 51, 1477-1508

27. LAm K.Y., Hung K.C., Chow S.T., 1989, Vibration analysis of plates with cutouts by the modified Rayleigh-Ritz method, Applied Acoustics, 28, 49-60

28. Lebedeva I.V., Knizhnik A.A., Popov A.M., Lozovik Yu. E., Potapkin B.V., 2012, Modeling of graphene-based NEMS, Physica E, 44, 949-954

29. Li C., Chou T.-W., 2003, A structural mechanics approach for the analysis of carbon nanotubes, International Journal of Solids and Structures, 40, 2487-2499

30. Li L., Hu Y., 2016, Wave propagation in fluid-conveying viscoelastic carbon nanotubes based on nonlocal strain gradient theory, Computational Materials Science, 112, 282-288

31. Liew K.M., Kitipornchai S., Leung A.Y.T., Lim C.W., 2003, Analysis of the free vibration of rectangular plates with central cut-outs using the discrete Ritz method, International Journal of Mechanical Sciences, 45, 941-959

32. Malekzadeh P., Bahranifard F., Ziaee S., 2013, Three-dimensional free vibration analysis of functionally graded cylindrical panels with cut-out using Chebyshev-Ritz method, Composite Structures, 105, 1-13

33. Metrikine A.V., Askes H., 2002, One-dimensional dynamically consistent gradient elasticity models derived from a discrete microstructure. Part 1: Generic formulation, European Journal of Mechanics A/Solids, 21, 555-572

34. Metrikine A.V., Askes H., 2006, An isotropic dynamically consistent gradient elasticity model derived from a 2D lattice, Philosophical Magazine, 86, 3259-3286 
35. Mindlin R., 1964, Micro-structure in linear elasticity, Archive for Rational Mechanics and Analysis, 16, 52-78

36. Mindlin R.D., Tiersten H.F., 1962, Effects of couple-stresses in linear elasticity, Archive for Rational Mechanics and Analysis, 11, 415-448

37. Mohammadi M., Farajpour A., Goodarzi, Dinari F., 2014, Thermo-mechanical vibration analysis of annular and circular graphene sheet embedded in an elastic medium, Latin American Journal of Solids and Structures, 11, 659-682

38. Murmu T., Pradhana S.C., 2009, Vibration analysis of nano-single-layered graphene sheets embedded in elastic medium based on nonlocal elasticity theory, Journal of Applied Physics, 105, 064319

39. Rahmani O., Pedram O., 2014, Analysis and modeling the size effect on vibration of functionally graded nano-beams based on nonlocal Timoshenko beam theory, International Journal of Engineering Science, 77, 55-70

40. Rajamani A., Prabhakaran R., 1977, Dynamic response of composite plates with cut-outs. Part 1: Simply-supported plates, Journal of Sound and Vibration, 54, 549-564

41. Reddy J.N., 1999, Theory and Analysis of Elastic Plates, Taylor and Francis, USA

42. REDDy J.N., 2011, Microstructure-dependent couple stress theories of functionally graded beams, Journal of Mechanics and Physics of Solids, 59, 2382-2399

43. Sadeghi M., Naghdabadi R., 2010, Nonlinear vibrational analysis of single-layered graphene sheets, Nanotechnology, 21, 105705-105710

44. Sakhaee-pour A., Ahmadian M.T., Naghdabadi R., 2008, Vibrational analysis of singlelayered graphene sheets, Nanotechnology, 19, 85702-85707

45. Scarpa F., Adhikari S., Phani A.S., 2009, Effective elastic mechanical properties of single layer graphene sheets, Nanothecnology, 20, 065709

46. Shen L., Shen H.-S., ZhAng C.-L., 2010, Nonlocal plate model for nonlinear vibration of single layer graphene sheets in thermal environments, Computational Materials Science, 48, 680-685

47. ŞıMŞEK M., 2016, Nonlinear free vibration of a functionally graded nanobeam using nonlocal strain gradient theory and a novel Hamiltonian approach, International Journal of Engineering Science, 105, $12-27$

48. Tham L.G., Chan A.H.C., Cheung Y.K., 1986, Free vibration and buckling analysis of plates by negative stiffness method, Composite Structures, 22, 687-692

49. Wang C.G., Lan L., Liu Y.P., TAn H.F., He X.D., 2013, Vibration characteristics of wrinkled single-layered graphene sheets, International Journal of Solids and Structures, 50, 1812-1823

50. Yang F., Chong A.C., Lam D.C.C., Tong P., 2002, Couple stress based strain gradient theory for elasticity, International Journal of Solids and Structurs, 39, 2731-2743

51. Zandiatashbar A., Lee G.-H., An S.J., Lee S., Mathew N., Terrones M., Hayashi T., Picu C.R., Hone J., Koratkar N., 2014, Effect of defects on the intrinsic strength and stiffness of graphene, Nature Communications, 5, 3186, DOI: 10.1038/ncomms4186 